\title{
Estimation of Relation between Financial Variables and Gold Price Using Fuzzy Multiple Linear Regression
}

\author{
B. Pushpa ${ }^{1}$, Dr. S. Muruganandam ${ }^{2}$ \\ ${ }^{I}$ (Department of Mathematics, Panimalar Institute of Technology, Poonamallee, Chennai, India) \\ (Research Scholar, Manonmaniam Sundaranar University, Tirunelveli,India ) \\ ${ }^{2}$ (Professor \& Head, Department of Mathematics, TRP Engineering College, Trichy, India)
}

\begin{abstract}
Price forecasting is an integral part of economic decision making. Forecasts may be used in numerous ways; specifically, individuals may use forecasts to try to earn income from speculative activities, to determine optimal government policies or to make business decisions. The importance of this topic is caused by instability in the world economy and stock markets; there is a growing interest in gold from investors and the current bull market of gold. It is believed that gold can be used to predict, to some degree, the stock market trend. However, the current forecasting algorithm offers poor precision in solutions for non-linear problems. In India, gold is one of the foundation assets for Indian households in the form of investment. It is viewed as secure and liquid investment. In this paper, a forecasting model for gold price using Multiple fuzzy linear regression using shape preserving operations were proposed with an extended objective function
\end{abstract}

.Keywords: Fuzzy linear regression, linear programming, Gold price, Financial variables

\section{Introduction}

Gold is a precious metal with which mankind had a long and illustrious relation and continues to do so. Gold is one of the most important commodities in the world. It is only the commodity that retains its value during all the periods of crises like economics, financial or political. As long as the world economy remains uncertain and investors feel inflation and sovereign default, gold will keep its allure (The Economist - July 8, 2010). The price of gold has been rising day by day, the fear of the world economy has caused the price of the gold to roar. Price of gold cannot be controlled but however it can be measured and forecasted for future decisions. Forecasting gold price is becoming increasingly important. For long in history, gold has been traded actively on international markets. Gold becomes an important tool for risk hedging as well as an investment avenue. In India, gold is one of the foundation assets for Indian households in the form of investment. It is viewed as secure, liquid investment. Gold served as money until other forms of currency were devised and even now gold is bought as an investment. For the prospective buyer of gold, it is important to know what all factors affect the rates of gold. This will allow a person to predict with good accuracy the trends in the rates and thus be able to direct an investment to more profit. Therefore, predicting the price of gold has become very significant and important to investors.

Sherman [1] concluded that gold price has significant positive relationship with unexpected inflation. Similar study was carried out by Moore (1990) that a gold price movement is predicted by a leading indicator of inflation. A research study by Larson and McQueen (1995) concluded that the coefficient of unexpected inflation for gold price is significant. Sadorsky (1999) explored that the correlation among three -month US treasury interest rates, $\mathrm{S} \& \mathrm{P} 500$ stock returns and oil prices.

Adrangi et al. (2003) concluded that gold has a positive relationship with the expected inflation; there exists no relationship with unexpected inflation. Ratanapalorn and Sharma (2007) studied the long term and short term relationships among the US stock price index (S\&P500) and macro economics variables during a study period from $1^{\text {st }}$ quarter 1975 to $4^{\text {th }}$ quarter 1998 . The stock price index and long term interest rates are negatively correlated as per the empirical study. Blose (2010) concluded that unexpected changes in the CPI do not affect gold spot prices and investors cannot determine market inflation expectations by examining the price of gold. The research work carried out by Devdutt pattanaik (2010)in his research paper "sacred gold" published by World Gold council mainly focuses on the mythological and cultural significance of gold in India. According to him, Indians love gold and this has been explained logically and culturally. Logically, gold is a tangible investment, unlike shares and bonds, a portable investment, unlike property and a beautiful ornament, one that can be worn daily as jewellery. But the same can be said of diamonds and other precious metals.

Kannan (2010) carried out an "econometric analysis" for knowing the Indian gold demand from 19802009 and the key drivers for this gold demand published by the World Gold Council. He had observed that for the higher income level group's platinum is a substitute for gold but for the majority gold is "a way of life". The value of gold stems from jewellery to a major vehicle of wealth accumulation by the low and middle-income households in rural and urban areas. The key drivers for the gold demand were real income level of the 
population, expectation of higher gold price, exchange rates, as an alternative instrument of saving, gold as an equity security, constrained supply conditions, the relationship between savings and uncertainty. Empirical studies reveal that gold demand is not only price sensitive but also affected by macro economic variables and financial variables. It has been understood from above that the factors affecting gold price also includes exchange rates. This area was further studied by Capie et. al(2005) and he concluded Gold as a Hedge against the US Dollar. In this study, a forecasting model for gold price using Multiple fuzzy linear regression using shape preserving operations were discussed and in India the relationship between gold price and other financial variables was studied over the period 2000-2010.

\section{Factors Influencing Price Of Gold}

The variables which influence the price of gold have a strong distinction between them, because of currency change or demand change due to inflation.

The first factor is the value of the US Dollar. It is an important question that is there any correlation between gold prices and the value of US dollar. The answer depends upon situation and changes with change in global economic scenario. Now there is inverse relationship between gold prices and US dollar. Since the dollar is the currency that most people have close relation in any fall in its value will lead to the prices of gold shooting up. The gold rate always had this relationship with the Dollar ever since the dollar became the global trading currency. As a tool of hedge, gold is demanded more than US dollar. When the price of gold depreciates the investors outside US will be benefited because the dollar price of the gold will increase.

The second factor is Gross domestic product growth rate (GDP growth rate). In India, the growth rate in GDP measures the change in the seasonally adjusted value of the goods and services produced by the Indian economy during the quarter. Quarterly data for the period 2005-11 shows that the growth rate of "real" GDP foresees the trend of the ensuing gold prices. This trend is consistent across India, China, and the US - three of the top ten gold consumers in the world. The trend shows that the gold price increases with the growth rate of GDP. This trend is evident for all the three countries for most quarters. One of the possible explanations for this trend is that when consumer's purchasing power increases, the demand for gold also raises, which inturn improves gold prices up, and vice-versa. Since the production of gold is time-consuming, the effect on gold prices occurs with a lag.

The third factor is the prime lending rate. In India, the prime lending rate is the average rate of interest charged on loans by commercial banks to private individuals and companies. Whenever interest rates fall gold prices increase and when inflation is on the rise so the gold prices also increase. Gold's most natural relationship to the general price level is what one might expect for any good or asset.

The fourth factor is Exchange reserve in India. In India, Foreign Exchange Reserves are the foreign assets held or controlled by the country central bank. The reserves are made of gold or a specific currency. They can also be special drawing rights and marketable securities denominated in foreign currencies like treasury bills, government bonds, corporate bonds and equities and foreign currency loans. Gold is one of the most widely discussed metals due to its prominent role in both the investment and consumer world. Even though gold is no longer used as a primary form of currency in developed nations, it continues to have a strong impact on the value of those currencies. Moreover, there is a strong correlation between its value and the strength of currencies trading on foreign exchanges.

The fifth factor which influences the gold price is inflation. Gold is also commonly believed to be a hedge against inflation. Researchers define inflation as the general rise in the price level (rather than an increase in the money supply) and use changes in the Consumer Price Index as the measure of monthly inflation. To be a hedge against inflation as the idea is most commonly understood, gold would not only have to be uncorrelated with inflation, it would have to be negatively correlated (Fei and Adibe 2010). Therefore, gold has always been considered as a good hedging instrument against inflation. Whenever interest rates fall gold prices increase and when inflation is on the rise so the gold prices also increase. A closer relationship exists between gold prices and inflation, that is, the rate of change in the general price level. In figure 3 clarifies the relationship by plotting the CPI inflation rate of ten year against the gold prices. By the help of a decade periods particularly stand out that higher gold prices also lead to the rate of inflation with reference to Indian country. A change of gold price has direct affect to inflation. Higher inflation seems to generate more demand of holding gold. Indirectly, gold is a fundamental input of many products and gold is no exception for this. Generally and easily, transportation cost is computed and included cost of all goods. In periods of inflation the value of money is decreasing. The definition of inflation is given by (http://www.investorwords.com Feb. 2011); "The overall general upward price movement of goods and services in an economy (often caused by an increase in the supply of money), usually as measured by the Consumer Price Index and the Producer Price Index. Over time, as the cost of goods and services increase, the value of a dollar is going to fall because a person won't be able to purchase as much with that dollar as he/she previously could". The effect of the decreasing value of the dollar is that people tend to start 
investing in gold as the value of gold is not changing as much as the value of the dollar, this is the stability of gold which have been discussed. The increased demand for gold will again push gold prices upward.

The sixth factor which affects the gold price is money supply. The money supply can include cash, coins and balances held in checking and savings accounts. Economists analyze the money supply and develop policies revolving around it through controlling interest rates and increasing or decreasing the amount of money flowing in the economy.

Keeping these factors in mind, an investor will be able to invest shrewdly. This will allow an investor to also know when to keep an investment and when to lose it.

\section{Basics On Fuzzy Linear Regression}

Fuzzy linear regression is a powerful tool to derive the fuzzy linear function for variables with uncertain nature. To derive the best fuzzy linear function between input and output variables, a model fitting measurement has to be defined. Model fitting measurement is an essential tool to measure the goodness of a defined model where the similarity between each of predicted and estimated outputs is computed. The weakest t-norm $\left(T_{\mathrm{w}}\right)$ based novel fuzzy regression model is developed using the concept of symmetric difference and also with Hausdorff distance. The proposed model will be useful in a realistic environment and improve upon the traditional fuzzy regression. The traditional system usually adopts alpha cut operations for its calculations. Here the $T_{w}$ - norm based operations are used to reduce the width of the estimated responses which will give exact prediction. Two characters were observed in $\mathrm{T}_{\mathrm{w}}$ norm arithmetic operations. First, it is well known that the addition/subtraction of fuzzy numbers by $\mathrm{T}_{\mathrm{w}}$ norm preserves the original shape of fuzzy numbers. For given shapes, in multiplication the $T_{w}$ norm preserves the original fuzzy numbers shape. Second, the $T_{w}$ norm operations can get more exact performance, which means smaller fuzzy spreads, under uncertain environment. This exact performance may successfully reduce accumulating phenomenon of fuzziness in complexity of fuzzy systems.

Fuzzy linear regression analysis can be seen as an optimization problem where the aim is to derive a model which fits the given dataset.

Consider the set of observed data $\left\{\left(\tilde{X}_{i}, \tilde{Y}_{i}\right), i=1, \ldots, n\right\}$ where $\tilde{X}_{i}$ is the explanatory variable which may be with crisp/ fuzzy numbers and $\tilde{Y}_{i}$ is the response variable which is either with symmetric/non-symmetric triangular or trapezoidal fuzzy numbers. Our aim is to fit a fuzzy regression model with fuzzy coefficients to the aforementioned data sets as follows: (D.H.Hong et al , 2001)

$\hat{\tilde{Y}}_{i}=\tilde{A}_{0} \oplus_{w}\left(\tilde{A}_{1} \otimes_{w} \tilde{X}_{i 1}\right) \oplus_{w} \ldots . . \oplus_{w}\left(\tilde{A}_{k} \otimes_{w} \tilde{X}_{i k}\right)=\tilde{A} \otimes_{w} \tilde{X}_{i}, \boldsymbol{i}=1, \ldots . \boldsymbol{n}$

where $\tilde{A}_{j}=\left(a_{j}, \alpha_{j}\right), j=1, \ldots k$ are fuzzy numbers and the arithmetic operations are based on the weakest $\mathrm{T}_{\mathrm{w}}$ norm. In this section, an extended objective function based on the symmetric difference along with similarity measure between two fuzzy sets, the observed and estimated values of the outputs using $T_{w}$ norm is proposed in which the coefficients of the models are also considered as fuzzy numbers. In the fuzzy linear regression, in order to estimate the model's parameter (fuzzy coefficient of the model) based on the sets of observations, some criteria should be considered. (1)The fuzzy output $\hat{\tilde{Y}}_{i}=\left(a^{T} x_{i}, \max _{1 \leq \mathrm{j} \leqslant k}\left(\left|a_{\mathrm{j}}\right| \gamma_{\mathrm{ij}}{ }^{\prime}\left|x_{\mathrm{ij}}\right| \alpha_{\mathrm{j}}\right)\right)$ ), for all the values of $(\mathrm{j}=1, \ldots \mathrm{k}), \hat{\tilde{Y}}_{i}$ - the estimated response is with atleast ' $\mathrm{h}$ ' degree of membership, i.e. $\mu\left(\hat{\tilde{Y}}_{i}\right) \geq h, j=1,2, . .,, m$. In order to obtain the best model, the value $\mathrm{h}$ is selected by the decision makers and it is the fitting degree of estimated linear regression model to a set of data. In addition, the parameter $\mathrm{h}$ can be defined as model's validity and certainty level. (2) The error could be minimized by minimizing the deviation between the observed and estimated responses.

To show the fitness (performance) of the fuzzy linear regression model, we compare the fuzzy estimated response of the model $\tilde{\tilde{Y}}_{i}$ with the observed one $\tilde{Y}_{i}$ where ' $i$ ' is the index of the data. There are different measures to determine the similarity between two fuzzy numbers. The similarity measure based on graded mean integration representation (Hsieh and Chen et al. (1999)) gives more accurate results. The similarity measure is just for comparison purposes, and any other type of objective function can be designed and then applied.

$$
S\left(\tilde{Y}_{i}, \tilde{\tilde{Y}}_{i}\right)=\frac{1}{1+A B S\left(a^{T} x_{i}-y_{i}\right)}
$$


The similarity measure varies between 0 and 1 , so the closer the value to 1 , the better the model. However for the sake of conformity, the dissimilarity measure $\left[1-S\left(\tilde{Y}_{i}, \tilde{\tilde{Y}}_{i}\right)\right]$ is considered and the value closer to 0 , the better the model.

Minimize $d\left(\tilde{Y}_{i}, \hat{\tilde{Y}}_{i}\right)$ using least absolute deviation (LAD) method,

Subject to

$$
\begin{aligned}
& \left|y_{i}-\sum_{1}^{k} a_{j} x_{i j}\right| \leq\left|L^{-1}(h)\right| \max _{1 \leq j \leq k}\left(\left|a_{j}\right| \gamma_{i j},\left|x_{i j}\right| \alpha_{j}\right)-\left|L^{-1}(h)\right| e_{i} \\
& \max _{1 \leq j \leq k}\left(\left|a_{j}\right| \gamma_{i j},\left|x_{i j}\right| \alpha_{j}\right) \geq 0, i=1, \ldots . n, \quad j=1, \ldots, k
\end{aligned}
$$

By considering the objective function and taking into all the constraints, the objective function is minimized with the use of linear programming method. The LINGO software is used for this purpose.

The LAD methods such as simple symmetric difference and Hausdorff distance, deals with the distance between the observed and estimated responses, which are shown in equations (6.4) and (6.5) respectively

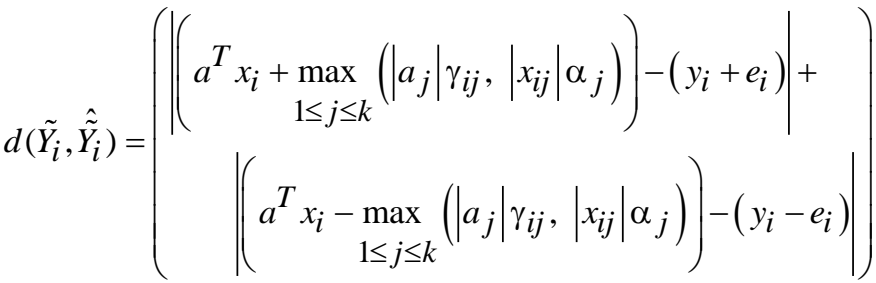

$$
\begin{aligned}
& d\left(\tilde{Y}_{i}, \hat{\tilde{Y}}_{i}\right)=\left(\left|y_{i}-a^{T} x_{i}\right|+0.5\left|e_{i}-\max _{1 \leq j \leq k}\left(\left|a_{j}\right| \gamma_{i j},\left|x_{i j}\right| \alpha_{j}\right)\right|\right)
\end{aligned}
$$

The extended objective function with the above defined LAD methods given in equation (1.4) and (1.5) along with the dissimilarity measure between the observed and estimated responses are in equations (1.6) and (1.7).

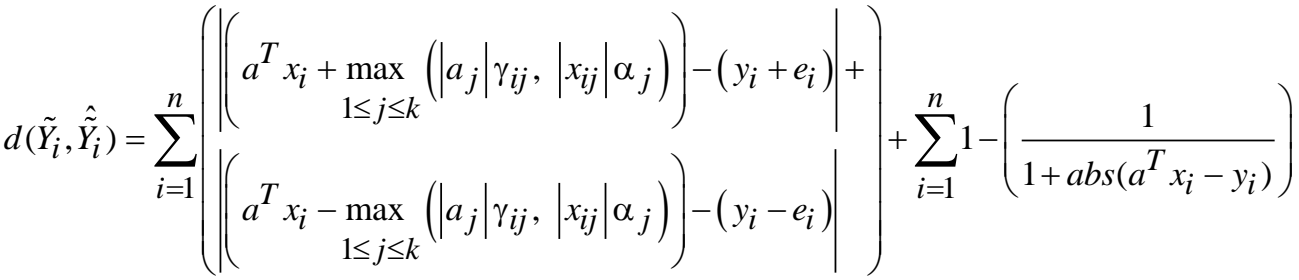

$$
\begin{aligned}
& d\left(\tilde{Y}_{i}, \hat{\tilde{Y}}_{i}\right)=\sum_{i=1}^{n}\left(\left|y_{i}-a^{T} x_{i}\right|+0.5\left|e_{i}-\max _{1 \leq j \leq k}\left(\left|a_{j}\right| \gamma_{i j},\left|x_{i j}\right| \alpha_{j}\right)\right|\right)+\sum_{i=1}^{n} 1-\left(\frac{1}{1+a b s\left(a^{T} x_{i}-y_{i}\right)}\right)
\end{aligned}
$$

Solving the optimization problem given in equation (3)using the objective functions given in equations (1.6) and (1.7), the fuzzy parameters are estimated using LINGO 13.0 (2004) software and the results are compared.

\section{Forecasting Model Of Gold Price}

The gold prices are time series data which are fixed twice a day in London. Factors influencing gold prices are many, but it is necessary to be selective in this study to ensure that the model developed is effective. The data used in this study are the Gold prices (GP) from the London PM fix (noon fixing time). GP is a dependent variable in this model.

The factors which influence the gold prices are US dollar exchange rate (USINR), Gross domestic product growth rate (GDP), Prime lending rate (LEND), Foreign exchange reserves, (FOREX), Inflation rate (INF) and Money supply (MS). Note these are not the only factors influencing the gold prices. These factors were used as independent variables in the multiple fuzzy linear regression models. The data used in this study are collected from www.tradingeconomics.com. In order to calculate GP, the related data for a period of 3 months for 10 years (2000 to 2010) has been extracted, so that the total number of each variable is equal to 30 .

The correlation coefficient between the dependent variable (GP) gold price and the independent variables USDINR, GDP, LEND, FOREX, INFL and MS are given in the following Table 6.1. 
Table 1.1 Correlation coefficient between the dependent variable (GP) gold price and the independent variables USDINR, GDP, LEND, FOREX, INFL and MS

\begin{tabular}{|l|l|l|l|l|l|l|}
\hline$\rho$ & USDINR & GDP & LEND & FOREX & INFL & MS \\
\hline GP & -0.28494 & 0.153617 & 0.124876 & 0.96959 & 0.827745 & 0.989868 \\
\hline
\end{tabular}

USDINR is negatively correlated with GP. GDP and LEND have very less correlation coefficient value and FOREX, INFL and MS are highly correlated with GP.

The aim of this study is to fit a fuzzy regression model using the shape preserving operations of the form:

$$
Y=\tilde{A}_{0} \oplus_{w} \tilde{A}_{1} \otimes_{w} X_{1} \oplus_{w} \tilde{A}_{3} \otimes_{w} X_{3} \oplus_{w} \tilde{A}_{4} \otimes_{w} X_{4} \oplus_{w} \tilde{A}_{5} \otimes_{w} X_{5} \oplus_{w} \tilde{A}_{6} \otimes_{w} X_{6}
$$

where each $X_{\mathrm{i}}$ is independent variable with crisp value and each coefficient $\tilde{A}_{i}$ is a triangular fuzzy number. Let us denote the independent variables as follows:

Y- GP; $\mathrm{X}_{1}$ - USDINR; $\mathrm{X}_{2}$ - GDP; $\mathrm{X}_{3}$ - LEND; $\mathrm{X}_{4}$ - FOREX; $\mathrm{X}_{5}$ - INFL; $\mathrm{X}_{6}-\mathrm{MS}$

TABLE 1.2 Fitting results of the relationship between the financial variables and gold price by fuzzy linear regression method using $T_{w}$ norm based operations with extended and simple symmetric difference based objective functions

\begin{tabular}{|c|c|c|}
\hline \multicolumn{2}{|c|}{ Using Extended objective function with symmetric difference } & \multirow{2}{*}{$\begin{array}{l}\text { Using objective function with symmetric difference } \\
d=921.0611\end{array}$} \\
\hline $\begin{array}{l}\text { Minimized } \\
\text { value of } \\
\text { objective } \\
\text { function } \\
\text { with ' } h \text { ' }\end{array}$ & $\begin{array}{l}\mathrm{d}=651.954 \\
\mathrm{~h}=0.127 \text { (34 iterations) }\end{array}$ & \\
\hline $\begin{array}{l}\text { Estimated } \\
\text { FLR } \\
\text { equation }\end{array}$ & $\begin{array}{r}\hat{\tilde{Y}}_{i}=(0,1.23456) \oplus_{w}(0,0.3908) \otimes_{w} X_{1} \oplus_{w}(0,1.23456) \otimes_{w} X_{2} \\
\oplus_{w}(2.98125,1.23456) \otimes_{w} X_{3} \oplus_{w}(0.01525,0) \otimes_{w} X_{4} \\
\oplus_{w}(13.43137,1.23456) \otimes_{w} X_{5} \oplus_{w}(0.45498,0.063115) \otimes_{w}\end{array}$ & $\begin{aligned} & \hat{\tilde{Y}}_{i}=(0,1.23456) \oplus_{w}(0,0) \otimes_{w} X_{1} \oplus_{w}(0,1.23456) \otimes_{w} X_{2} \\
& \oplus_{w}(2.98125,1.23456) \otimes_{w} X_{3} \oplus_{w}(0.01525,0) \otimes_{w} X_{4} \\
& X_{6} \quad \oplus_{w}(13.43137,1.23456) \otimes_{w} X_{5} \oplus_{w}(0.45498,0.061215)\end{aligned}$ \\
\hline MAPE & 0.059378 & 0.05937 \\
\hline $\begin{array}{l}\text { Goodness of } \\
\text { fit measure }\end{array}$ & 0.619087 & 0.60329 \\
\hline RIC & 0.060615 & 0.060602 \\
\hline
\end{tabular}

This study examines the relationship between dependent variable (GP) and independent variables (USDINR, GDP, LEND, FOREX, INFL and MS) through fuzzy linear regression method using $\mathrm{T}_{\mathrm{w}}$ norm based operations. The fitting results of the relationship between financial variables and gold price by fuzzy linear regression method using $\mathrm{T}_{\mathrm{w}}$ norm based operations is summarized in the following Tables 1.2 and 1.3. The estimated regression model is evaluated with goodness of fit measure $(\mathrm{Xu}$ and $\mathrm{Li}, 2001)$ and MAPE values.

TABLE 1.3 Fitting results of the relationship between the financial variables and gold price by fuzzy linear regression method using $T_{w}$ norm based operations with extended and simple Hausdorff distance based objective functions

\begin{tabular}{|c|c|c|}
\hline \multicolumn{2}{|c|}{ Using Extended objective function with Hausdorff distance } & \multirow{2}{*}{$\begin{array}{l}\text { Using objective function with Hausdorff distance } \\
\qquad \begin{array}{l}\mathrm{d}=8743 \\
\mathrm{~h}=0.976 \\
(16 \text { iterations })\end{array}\end{array}$} \\
\hline $\begin{array}{l}\text { Minimized } \\
\text { value of } \\
\text { objective } \\
\text { function with } \\
\text { 'h' }\end{array}$ & $\begin{array}{l}d=246.74 \\
h=0.16 \\
\text { (33 iterations) }\end{array}$ & \\
\hline $\begin{array}{l}\text { Estimated } \\
\text { FLR equation }\end{array}$ & $\begin{aligned} \hat{\tilde{Y}}_{i}= & (0,1.23456) \oplus_{w}(0,0) \otimes_{w} X_{1} \oplus_{w}(0,1.23456) \otimes_{w} X_{2} \\
& \oplus_{w}(2.98125,1.23456) \otimes_{w} X_{3} \oplus_{w}(0.01525,0) \otimes_{w} X_{4} \\
& \oplus_{w}(13.4314,1.23456) \otimes_{w} X_{5} \oplus_{w} \\
& (0.45498,0.06559) \otimes_{w} X_{6}\end{aligned}$ & $\begin{aligned} \hat{\tilde{Y}}= & (0,1.23456) \oplus_{w}(0,1.23456) \otimes_{w} X_{1} \oplus_{w}(0,1.23456) \otimes_{w} X_{2} \\
& \oplus_{w}(7.8455,1.23456) \otimes_{w} X_{3} \oplus_{w}(0.02824,0.2609) \otimes_{w} X_{4} \\
& \oplus_{w}(5.92368,1.23456) \otimes_{w} X_{5} \oplus_{w}(0.33057,1.23456) \otimes_{w} X_{6}\end{aligned}$ \\
\hline MAPE & 0.059373 & 0.05544 \\
\hline $\begin{array}{l}\text { Goodness of } \\
\text { fit measure }\end{array}$ & 0.640462 & 0.99958 \\
\hline RIC & 0.0606 & 0.073108 \\
\hline
\end{tabular}

As it can be observed in Tables 1.2 and 1.3, the calculated parameters in fuzzy regression model are triangular fuzzy numbers which include the central value, the degree of ambiguity and their possible uncertainty. 
Also from the above tables, it is clear that the fuzzy linear regression model using $T_{w}$ norm with extended objective function gives minimum objective value i.e. minimum least absolute deviation between the observed and estimated ones than the usual simple least absolute deviation based objective function.

The estimated gold prices along with the actual gold prices are compared in the following Figures. Actual gold price is represented in continuous curve and the predicted gold price is in continuous straight line.

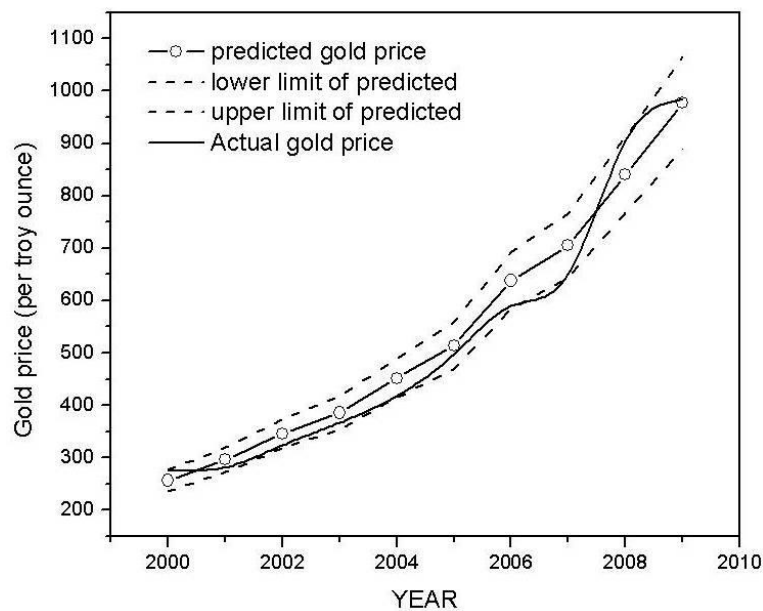

Fig.1.1Estimated fuzzy linear regression model using symmetric difference between the estimated and the observed using $T_{w}$ norm based operations.

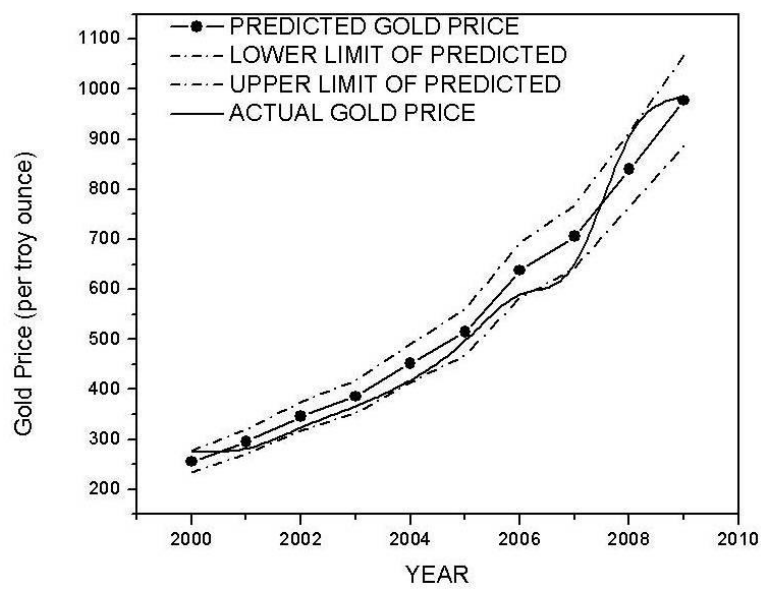

Fig. 1.2 Estimated fuzzy linear regression model with the extended objective with symmetric difference and similarity measure using the $T_{w}$ norm operations

The Figure 1.1 reveals that the fitted trend line has $60 \%$ goodness of fit measure with the observed.

From the Figure 1.2, it is evident that using the extended objective function with symmetric difference, the estimated gold price trend line has $62 \%$ goodness of fit measure with the actual gold price.

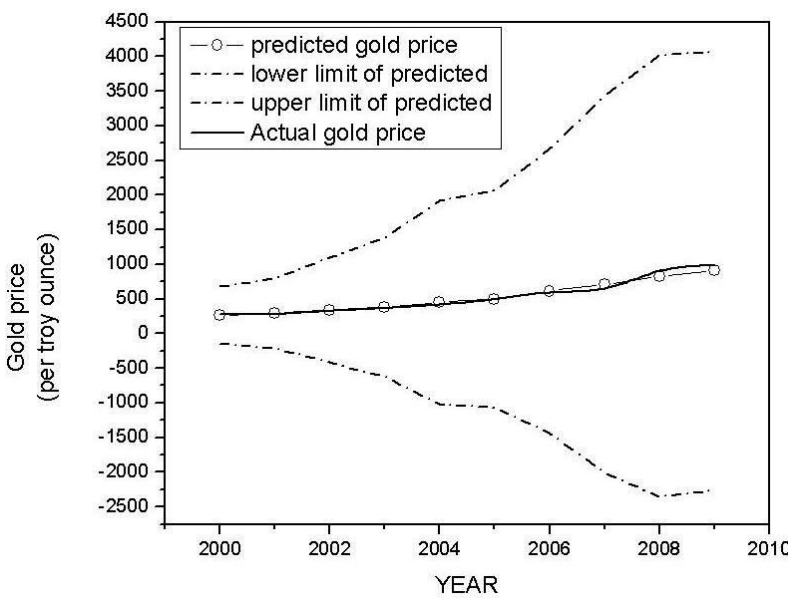

Fig. 1.3Estimated fuzzy linear regression model with minimizing the Hausdorff distance between the estimated and the observed using $\mathbf{T}_{\mathrm{w}}$ norm based operations

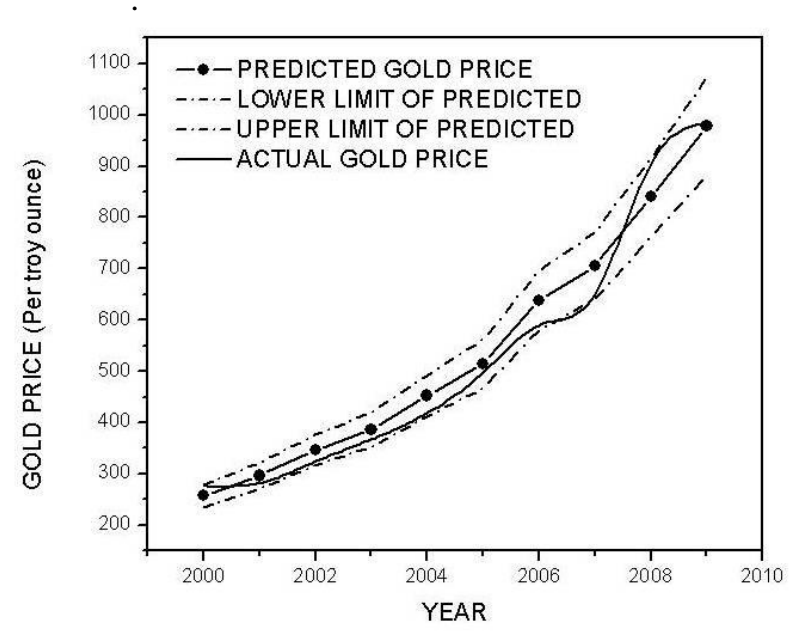

Fig. 1.4 Estimated fuzzy linear regression model with the extended objective with Hausdorff distance and similarity measure using the $T_{w}$ norm operations

The Figure 1.3 indicates the trend line through the centers of the estimated has $99 \%$ goodness of fit measure. Even though the goodness of fitness is high, the simple Hausdorff distance based LAD method gives a wide range of estimated spreads and the estimated centers coincides with observed centers

From the Figur.1.4, it is obvious that the estimated trend line has $64 \%$ goodness of fit measure with the actual gold price. The figures 1.1 to 1.4 reveal that the estimated interval result with very narrow spread is due to the shape preserving operations. Also in the objective function by minimizing the dissimilarity and LAD, yields the effective performance of shape preserving operations. 


\section{Conclusion}

Many accounting problem comprises the financial variables and relationships which are difficult to measure accurately. In order to develop a fuzzy linear regression model, London PM fix for the gold price is used as the dependent variable. In the study of the relationship between financial variables (USDINR, GDP, LEND, FOREX, INF and MS) and gold price, this structure can be considered as a fuzzy linear function in which the parameter are determined by fuzzy set theory using $T_{w}$ norm based operations. The results of fitting the fuzzy regression model using $\mathrm{T}_{\mathrm{w}}$ norm suggests that there is a relationship between the financial variables and gold price.

From the coefficients, it is concluded that a decrease in US dollar rate may result in increase in the gold price. Similarly, a decrease in GDP growth rate increases the gold price. For Prime lending rate, Exchange reserve, inflation and money supply, a unit increase will result in the increase of gold price. The regression model that has explanatory variables as crisp numbers can be a good model only if the shape of the membership function for the fuzzy regression coefficient is identical with that of the response variable, i.e. the predicted values should coincide almost with the mode of the observed response. From the Figures 1.1, 1.2, 1.3 and 1.4, the predicted upper and lower limit values are with very small width from the center denotes the predicted models with extended objective function has effective performance in forecasting using the extended objective function with Hausdorff distance with $\mathrm{T}_{\mathrm{w}}$ norm based operations.

\section{References}

[1]. Sherman, E. J., “A gold pricing model”, Journal of Portfolio Management, 9, 1983, 68-70.

[2]. Moore, G. H., "Gold prices and a leading index of inflation", Challenge 33, 1990, 52-56.

[3]. Larsen, A. B., McQueen, G. R., "REITs, real estate, and inflation: Lessons from the gold market", Journal of Real Estate Finance \& Economics 10, 1995, 285-297.

[4]. Perry Sadorsky, "Oil price shocks and stock market activity", Energy Economics, 21(5), 1999, 449-469.

[5]. Adrangi, B., Chatrath, A. and Raffiee, K., "Economic activity, inflation, and hedging: The case of gold and silver investments", The Journal of Wealth Management 6, 2003, 60-77.

[6]. Orawan Ratanapakorn, Subhash, C. Sharma, "Dyanamic analysis between the US stock Returns and Macroeconomics variables", Applied Finance Economics, 17(5), 2007, 369-377.

[7]. Blose, L. D., "Gold prices, Cost of carry and expected inflation", Journal of Economics and Business, 62, $2010,35-47$.

[8]. Devdutt Pattanaik, "Sacred Gold", World Gold Council, November, 2010, 12-15.

[9]. Kannan. R, "An econometric analysis", World Gold Council, 2010, 16-27.

[10]. Capie, F., Terence, C.M. and Wood, G., "Gold as a hedge against the dollar", International Financial Markets, Institution and Money $15,2005,343-352$.

[11]. Fan Fei and Kelechi Adibe, "Theories of Gold Price Movements: Common Wisdom or Myths", Undergraduate Economic Review, 6(1), 2010, Article 5.

[12]. Hong, D. H., Lee, S. and Do, D.Y., "Fuzzy linear regression analysis for fuzzy input-output data using shape preserving operations", Fuzzy Sets and Systems, 122, 2001, 513-526.

[13]. Hsieh, C. H. and Chen, S. H., "Similarity of generalized fuzzy numbers with graded mean integration representation", in Proceedings of Eighth international fuzzy system association world congress, vol. 2, Taipei, Taiwan, Republic of China, 551-555, 1999.

[14]. Xu, R. and Li, C., "Multidimensional least- squares fitting with a fuzzy model", Fuzzy sets and systems, 119, 2001,.215-223. 